\title{
Treinamento intervalado de alta intensidade na ocorrência de lesões em corredores:
}

\section{Uma revisão da literatura}

\author{
High intensity interval training in the occurrence of injuries in runners: A literature review \\ Entrenamiento en intervalos de alta intensidad ante la ocurrencia de lesiones en corredores:
}

Revisión de la literatura

Recebido: 13/04/2021 | Revisado: 20/04/2021 | Aceito: 20/04/2021 | Publicado: 05/05/2021

\author{
Bruno Alvarenga Soares \\ ORCID: https://orcid.org/0000-0002-1709-3747 \\ Universidade Federal de Minas Gerais, Brasil \\ E-mail: brunoalvarengasoares@outlook.com \\ Vinícius Veiga de Souza \\ ORCID: https://orcid.org/0000-0002-4767-1624 \\ Universidade Federal de Minas Gerais, Brasil \\ E-mail: vinivsfisio@gmail.com \\ Vanessa Kelly da Silva Lage \\ ORCID: https://orcid.org/0000-0002-0381-5622 \\ Universidade Federal dos Vales do Jequitinhonha e Mucuri, Brasil \\ E-mail: vanessakellys1@hotmail.com
}

\begin{abstract}
Resumo
Introdução: Especula-se que uma modalidade tão intensa como o Treinamento Intervalado de Alta Intensidade poderia ser um dos fatores para gerar um maior número de lesões em corredores. Objetivo: Revisar, na literatura, estudos que registram lesões provocadas pelo treinamento intervalado de alta intensidade em praticantes recreativos ou profissionais de corrida. Metodologia: Trata-se de uma revisão da literatura utilizando as bases de dados LILACS (Literatu $\neg$ ra Latino-Americana e do Caribe em Ciências da Saúde), MEDLINE (US National Library of Medicine), SciELO (Scientific Eletronic Library Online) com os descritores "high intensity interval training", "injury", "running" e "runners" com suas variações. A pesquisa foi limitada com base na disponibilidade de texto (texto completo disponível), data de publicação (de janeiro de 2000 a março 2020), espécies (humanos), sem restrição de idioma. Foi permitida inclusão de qualquer modalidade de corredores (recreativos e profissionais). Foram excluídos da pesquisa os artigos que não utilizavam HIIT ou que não apresentavam relação entre treinamento e lesões. Resultados: Foram eleitos 32 artigos para a leitura do re $\neg$ sumo e excluídos os que não condiziam com o propósito deste estudo. Foram selecionados 7 artigos que preenchiam os critérios propostos e foram seguidos, nessa ordem, os seguintes passos: leitura exploratória; análise dos tex ᄀtos, finalizando com a realização de leitura interpretatiาva e redação. Conclusão: Ainda há poucos estudos que investigaram a ocorrência de lesões com a prática do treinamento intervalado de alta intensidade em corredores. Dos estudos encontrados, as amostras eram pequenas e se apresentavam em diferentes tipos de corredores como de elite e recreativos.
\end{abstract}

Palavras-chave: Treinamento intervalado de alta intensidade; Corredores; Lesões.

\begin{abstract}
Introduction: It is speculated that a modality as intense as the High Intensity Interval Training (HIIT) could be one of the factors to generate greater number of injuries in runners. Objective: To review, in the literature, studies that record injuries caused by HIIT in recreational or running professionals. Methodology: This is a literature review using the Latin American and Caribbean Literature in Health Sciences databases, National Library of Medicine and The National Institute of Health, US National Library of Medicine, Scientific Electronic Library Online with the descriptors "high intensity interval training", "injury", "running" and "runners" with their variations. The search was limited based on the availability of text (full text available), date of publication (from January 2000 to March 2020), species (humans), without language restriction. Inclusion of any type of runners (recreational and professional) was allowed. Articles that did not use HIIT or that had no relationship between training and injuries were excluded from the research. Results: 32 articles were chosen to read the summary and those that did not match the purpose of this study were excluded. Seven articles were selected that met the proposed criteria and the following steps were followed in that order: exploratory reading; analysis of the texts, ending with the performance of interpretative reading and writing. Conclusion: There are still few studies that investigated the occurrence of injuries with the practice of
\end{abstract}


HIIT in runners. The samples were small and presented different samples of the different running practices as elite and recreational runners.

Keywords: High intensity interval training; Runners; Injuries.

\section{Resumen}

Introducción: Se especula que una modalidad tan intensa como el Entrenamiento de Intervalos de Alta Intensidad (HIIT) podría ser uno de los factores para generar mayor número de lesiones en los corredores. Objetivo: Revisar, en la literatura, estudios que registran lesiones causadas por HIIT en profesionales recreativos o del running. Metodología: Esta es una revisión de literatura utilizando las bases de datos de Literatura Latinoamericana y del Caribe en Ciencias de la Salud, Biblioteca Nacional de Medicina y el Instituto Nacional de Salud, Biblioteca Nacional de Medicina de EE. UU., Biblioteca Electrónica Científica en Línea con los descriptores "entrenamiento en intervalos de alta intensidad", "lesión", "correr" y "corredores" con sus variaciones. La búsqueda se limitó en función de la disponibilidad de texto (texto completo disponible), fecha de publicación (de enero de 2000 a marzo de 2020), especie (humanos), sin restricción de idioma. Se permitió la inclusión de cualquier tipo de corredores (recreativos y profesionales). Los artículos que no usaban HIIT o que no tenían relación entre entrenamiento y lesiones fueron excluidos de la investigación. Resultados: Se eligieron 32 artículos para leer el resumen y se excluyeron aquellos que no coincidían con el propósito de este estudio. Se seleccionaron siete artículos que cumplieron con los criterios propuestos y se siguieron los siguientes pasos en ese orden: lectura exploratoria; análisis de los textos, finalizando con la realización de lectura y escritura interpretativas. Conclusión: aún son pocos los estudios que investigaron la ocurrencia de lesiones con la práctica de HIIT en corredores. Las muestras fueron pequeñas y presentaron diferentes muestras de las diferentes prácticas de carrera como corredores de élite y recreativos.

Palabras clave: Entrenamiento por intervalos de alta intensidad; Corredores; Lesiones por correr.

\section{Introdução}

O Treino intervalado de alta intensidade ou High Intensity Interval Training (HIIT) é caracterizado por breves e repetidas sessões de exercício intenso, próximo a 100\% da capacidade máxima do indivíduo intercaladas por período de repouso ou exercício de baixa intensidade (Gibala \& McGee, 2008; Gillen \& Gibala, 2014). Tal modalidade promove adaptações semelhantes ao treino aeróbio de intensidade moderada (Bucci, Vinagre, Campos, Curi, \& Pithon-Curi, 2005), sendo considerado uma intervenção tempo eficiente para melhora do condicionamento aeróbio em poucas sessões de treinamento (Burgomaster, Hughes, Heigenhauser, Bradwell, \& Gibala, 2005; Little, Safdar, Wilkin, Tarnopolsky, \& Gibala, 2010).

O HIIT permite a manipulação de até nove variáveis, que incluem a duração e intensidade do treino intervalado, duração e intensidade do descanso, modalidade de exercício, número de repetições, número de séries, bem como a duração e intensidade da recuperação entre séries. A manipulação de qualquer uma dessas variáveis pode afetar as respostas fisiológicas agudas ao HIIT (Buchheit, \& Laursen, 2013).

Não existe um padrão de tempo de duração da atividade, tempo de cada estímulos e as intensidades adequadas (Schoenmakers \& Reed, 2019). A literatura apresenta diversos seguimentos para o HIIT: O SIT (Sprint Interval Training) que corresponde a sprints de 20 a 30 segundos intercalados por intervalos entre 2 a 4 minutos que podem ser ativos ou passivos, com esforços próximos ou superiores ao VO2 máx., O RST (Repeated Sprint Training) que são sprints repetidos com pequenos tiros de 3 a 7 segundos de duração e separados por pequenos períodos de recuperação (menores que 60 segundos). O HIIT com intervalos curtos são estímulos com intervalos de descanso menores ou iguais a 60 segundos e com intensidade mais baixa (60\% do VO2máx.). O HIIT com intervalos longos contém intervalos acima de 60 segundos de descanso entre os estímulos (Buchheit \& Laursen, 2013; MacInnis \& Gibala, 2017).

Especula-se que uma modalidade tão intensa como o HIIT poderia ser um dos fatores para gerar um maior número de lesões em corredores. Lesões são desordens do sistema musculoesquelético ou contusões. As lesões esportivas referem-se a uma variedade de danos musculoesqueléticos relacionados a participação no esporte. Lesões são uma barreira importante para essa prática. (Hespanhol Junior et al 2015). Estudos apontam que a incidência de lesões por ano atinge entre 40-50\% dos 
Research, Society and Development, v. 10, n. 5, e24310515008, 2021

(CC BY 4.0) | ISSN 2525-3409 | DOI: http://dx.doi.org/10.33448/rsd-v10i5.15008

corredores (García-Pinillos, Soto-Hermoso, \& Latorre-Román, 2017; Latorre-Román, García Pinillos, Bujalance-Moreno, \& Soto-Hermoso, 2017). As lesões são multifatoriais, mas geralmente as lesões na corrida, são atribuidas ao treinamento o que pode afastar corredores do esporte e das competições (García-Pinillos et al., 2017).

Lesões podem ser classificadas de acordo com sua magnitude (leve, média e grave), agudas ou crônicas, por uso excessivo (overuse), lesões recorrentes, relesão, exacerbação da lesão e podemos classificar a região da lesão. A utilização de alguns instrumentos de medição, como os questionários, auxilia a determinar a incidência ou a prevalência de lesões (Hespanhol Junior, Barboza, van Mechelen, \& Verhagen, 2015). Ainda, lesões causadas por overuse correspondem a aproximadamente $49 \%$ das lesões musculoesqueléticas, enquanto, as lesões agudas cerca de $13 \%$ e outras patologias cerca de 36\% (Clarsen, Rønsen, Myklebust, Flørenes, \& Bahr, 2014).

Clarsen et al. (2014) em seu estudo com atletas olímpicos e paraolímpicos de diferentes esportes, encontrou que lesões causadas por overuse correspondem a aproximadamente $49 \%$ das lesões musculoesqueléticas, enquanto, as lesões agudas cerca de $13 \%$ e outras patologias cerca de $36 \%$ (Clarsen et al 2014).

A carga de treinamento mais elevada, manipulando a intensidade, duração e frequência de treinamento, proporciona adaptações inflamatórias e de estresse oxidativo positivas (Freitas et al., 2019; Melo et al., 2019), além de adaptações cardiovasculares e neuromusculares (Bucci et al., 2005), porém, se essas variáveis forem acima do que o sistema tolera, lesões podem ocorrer (Bucci et al., 2005).

Diante do exposto e com o aumento da prática do HIIT nos treinos de corredores e o possível aumento de lesões que pode estar relacionado, essa revisão de literatura justifica-se pela necessidade de explorar o que a literatura atual traz sobre os riscos e benefícios dessa modalidade de treinamento quanto à presença de lesões em corredores para que possa ser traçado estratégias no treinamento de corredores. Sendo assim, os objetivos deste estudo são revisar, na literatura, estudos que registram lesões provocadas pelo treinamento intervalado de alta intensidade em praticantes recreativos ou profissionais de corrida e elucidar possíveis mecanismos de lesão provocados pelo HIIT em corredores. se o parágrafo como modelo.

\section{Metodologia}

Trata-se de uma revisão da literatura utilizando as bases de dados LILACS (Literatura Latino-Americana e do Caribe em Ciências da Saúde), MEDLINE (US National Library of Medicine), SciELO (Scientific Eletronic Library Online).

Os descritores utilizados foram "high intensity interval training", "injury", "running" e "runners" com suas variações. A pesquisa foi limitada com base na disponibilidade de texto (texto completo disponível), data de publicação (de janeiro de 2000 a março 2020), espécies (humanos), sem restrição de idioma. Foi permitida inclusão de qualquer modalidade de corredores (recreativos e profissionais). Foram excluídos da pesquisa os artigos que não utilizavam HIIT ou que não apresentavam relação entre treinamento e lesões.

\section{Resultados}

Foram eleitos 32 artigos para a leitura do resumo e excluídos os que não condiziam com o propósito deste estudo. Após a leitura dos resumos, foram selecionados 7 artigos que preenchiam os critérios propostos e foram seguidos, nessa ordem, os seguintes passos: leitura exploratória; análise dos textos, finalizando com a realização de leitura interpretativa e redação.

A partir da análise dos textos, foi elaborada o quadro abaixo (Quadro 1) que expõe os autores, amostra, objetivo, intervenção, instrumentos e resultados nos artigos selecionados com o objetivo de sistematizar e facilitar a comparação entre as abordagens das diversas fontes. 
Quadro 1. Análise descritiva dos estudos incluídos

\begin{tabular}{|c|c|c|c|c|}
\hline AUTORES & AMOSTRA & OBJETIVO & INTERVENÇÃO & RESULTADOS \\
\hline $\begin{array}{l}\text { GARCÍA- } \\
\text { PINILLOS et al } \\
2016\end{array}$ & $\begin{array}{l}\text { Estudo observacional com } \\
18 \text { adolescentes treinaram } \\
\text { recreativamente corrida } \\
\text { de resistência sendo } 16 \\
\text { homens e } 2 \text { mulheres com } \\
\text { idade de } 30.9 \pm 11.7 \text { anos. }\end{array}$ & $\begin{array}{l}\text { Avaliar as características cinemáticas da } \\
\text { corrida durante os estágios inicial e final } \\
\text { de dois protocolos de HIIT com carga } \\
\text { externa semelhante, mas com ritmo } \\
\text { médio de corrida diferente, bem como } \\
\text { comparar as alterações induzidas pela } \\
\text { fadiga durante os dois HIIT. }\end{array}$ & $\begin{array}{l}\text { Cada atleta foi testado em } 2 \text { ocasiões separadas } \\
\text { por } 7 \text { dias: } \\
\text { 1- } 10 \text { corridas de } 400 \text { m com } 90-120 \text { s de } \\
\text { recuperação entre corridas } 2-40 \text { corridas de } \\
100 \text { m com } 25-30 \text { s de recuperação entre } \\
\text { corridas. }\end{array}$ & $\begin{array}{l}\text { Constataram que a intensidade acima da velocidade } \\
\text { associada ao consumo máximo de oxigênio não } \\
\text { perturba consistentemente a cinemática da corrida } \\
\text { de corredores de resistência treinados. }\end{array}$ \\
\hline $\begin{array}{l}\text { GARCÍA- } \\
\text { PINILLOS et al } \\
2016\end{array}$ & $\begin{array}{l}\text { Estudo observacional com } \\
28 \text { corredores de } \\
\text { resistência do sexo } \\
\text { masculino com idade } \\
\text { média de } 27 \pm 7 \text { anos. }\end{array}$ & $\begin{array}{l}\text { Avaliar as características cinemáticas da } \\
\text { corrida durante os estágios inicial e final } \\
\text { que ocorrem durante uma sessão comum } \\
\text { de HIIT para corredores de endurance. }\end{array}$ & $\begin{array}{l}\text { Medidas repetidas em um único grupo, no qual } \\
\text { os autores tentam determinar as alterações } \\
\text { induzidas pela fadiga na cinemática da corrida } \\
\text { de corredores de endurance durante um } \\
\text { protocolo HIIT comum, Foram realizadas duas } \\
\text { análises de cluster, de acordo com o ritmo } \\
\text { médio de corrida - Mais Rápido vs. Mais Lento, } \\
\text { e de acordo com o nível de exaustão atingido - } \\
\text { Grupo esgotado vs. não esgotado (GE e NEG). }\end{array}$ & $\begin{array}{l}\text { Uma sessão HIIT comum para corredores de } \\
\text { endurance não perturba consistentemente ou } \\
\text { substancialmente a cinemática da corrida de } \\
\text { corredores masculinos treinados. } \\
\text { A maioria das lesões na corrida pode ser atribuída } \\
\text { ao uso excessivo de repetidos períodos de atividade. }\end{array}$ \\
\hline KOHN et al. 2011 & $\begin{array}{l}\text { Estudo experimental com } \\
18 \quad \text { corredores de } \\
\text { endurance treinados. }\end{array}$ & $\begin{array}{l}\text { Investigar as adaptações fisiológicas e } \\
\text { musculares esqueléticas em corredores de } \\
\text { endurance submetidos a } 6 \text { semanas de } \\
\text { HIIT. }\end{array}$ & $\begin{array}{l}\text { Realizados o protocolo de HIIT } 2 \text { vezes por } \\
\text { semana, durante } 6 \text { semanas. Testes de } \\
\text { exercício máximo e submáximo e biópsias } \\
\text { musculares foram realizadas antes e após o } \\
\text { treinamento. }\end{array}$ & $\begin{array}{l}\text { O HIIT no treinamento de resistência com } \\
\text { corredores, não causou adaptações na capacidade } \\
\text { oxidativa do músculo, mas aumentou a atividade do } \\
\text { metabolismo do lactato, especialmente nas fibras } \\
\text { tipo IIa e a relação com a velocidade absoluta no } \\
\text { HIIT. } \\
6 \text { semanas de HIIT provocou melhorias no } \\
\text { desempenho e aumento do metabolismo de lactato. }\end{array}$ \\
\hline
\end{tabular}




\begin{tabular}{|c|c|c|c|c|}
\hline $\begin{array}{l}\text { LATORRE- } \\
\text { ROMÁN et al } \\
2016\end{array}$ & $\begin{array}{l}\text { Estudo experimental com } \\
13 \text { atletas saudáveis, de } \\
\text { elite e altamente } \\
\text { treinados, do } \quad \text { sexo } \\
\text { masculino com idade } \\
\text { média de } 30,83 \pm 7,82 \\
\text { anos. }\end{array}$ & $\begin{array}{l}\text { Avaliar as características cinemáticas da } \\
\text { corrida e os padrões de pisada durante os } \\
\text { estágios iniciais e finais do treinamento } \\
\text { intermitente de alta intensidade real e } \\
\text { comum (HIIT): } 5 \times 2000 \mathrm{~m} \text { com } \\
\text { recuperação de } 120 \text { s entre as corridas. }\end{array}$ & $\begin{array}{l}\text { Os participantes realizaram cinco corridas de } \\
2000 \mathrm{~m} \text { com } 120 \mathrm{~s} \text { de recuperação entre as } \\
\text { sessões de corrida }(5 \times 2000 \mathrm{~m}) \text {. Foram } \\
\text { realizados protocolos HIIT, medindo } \\
\text { aproximadamente a velocidade associada ao } \\
\text { VO2máx, (medido através da velocidade de } \\
\text { uma corrida de } 3000 \mathrm{~m}) \text {. O desempenho e a } \\
\text { fadiga em cada corrida foram registrados } \\
\text { através do tempo gasto, pico da frequência } \\
\text { cardíaca e classificações de esforço percebido. } \\
\text { Foi realizada uma comparação dentro do grupo, } \\
\text { considerando-se as condições fatigadas e não } \\
\text { fatigadas no início e no final do protocolo, } \\
\text { respectivamente. }\end{array}$ & $\begin{array}{l}\text { O estudo não encontrou alterações nas } \\
\text { características cinemáticas e nos padrões de pisada } \\
\text { em corredores de resistência após fadiga induzida } \\
\text { por um HIIT, podendo indicar que treinadores e } \\
\text { corredores não devem temer efeitos prejudiciais } \\
\text { consideráveis do HIIT na cinemática da corrida e } \\
\text { no padrão de pisada e, portanto, na técnica de } \\
\text { corrida. }\end{array}$ \\
\hline $\begin{array}{l}\text { MUJIKA et al } \\
2000\end{array}$ & $\begin{array}{l}\text { Estudo experimental com } \\
8 \text { corredores masculinos } \\
\text { de média distância bem } \\
\text { treinados com idade } \\
\text { média de } 19,9 \pm 1,8 \text { anos }\end{array}$ & $\begin{array}{l}\text { Examinar algumas respostas fisiológicas } \\
\text { e de desempenho de um cone 6-d, e a } \\
\text { influência da intensidade e volume do } \\
\text { treinamento nessas respostas. }\end{array}$ & $\begin{array}{l}\text { Durante a semana } 16 \text { da temporada de inverno, } \\
\text { os participantes realizaram um dos dois } \\
\text { possíveis programas experimentais de redução. } \\
\text { Como o programa de treinamento estabelecido } \\
\text { pelo treinador seguiu uma estrutura de } \\
\text { mesociclo de } 4 \text { semanas, consistindo em } 3 \\
\text { semanas de treinamento de sobrecarga e } 1 \\
\text { semana de treinamento de recuperação, o } \\
\text { programa 6-taper de cada atleta foi estabelecido } \\
\text { em relação aos valores médios diários de } \\
\text { treinamento de } 3 \text { semanas antes ao cone. Um } \\
\text { dos afunilamentos (conicidade de volume } \\
\text { moderado, TVM) consistia em uma redução } \\
\text { progressiva da distância LICT e HIIT em até }\end{array}$ & $\begin{array}{l}\text { Alterações fisiológicas induzidas por conicidade em } \\
\text { corredores de média distância são principalmente } \\
\text { hematológicos, e mudanças fisiológicas distintas } \\
\text { são provocadas pelo LICT (treinamento contínuo de } \\
\text { baixa intensidade) e HIIT durante o afunilamento. } \\
\text { Corredores de média distância podem reduzir } \\
\text { progressivamente o volume habitual de treinamento } \\
\text { em pelo menos } 75 \% \text { durante uma redução de nível } \\
\text { 6-d. }\end{array}$ \\
\hline
\end{tabular}




\begin{tabular}{|c|c|c|c|c|}
\hline & & & $\begin{array}{l}50 \% \text { dos valores dos pré-afunilamentos }(90,80, \\
70,60,50 \text { e } 50 \% \text { durante os dias de } \\
\text { afunilamento } 1 \text { a } 6 \text {, respectivamente). No taper } \\
\text { de baixo volume (LVT), por outro lado, os } \\
\text { valores LICT e HIIT do pré-papel foram } \\
\text { reduzidos em } 75 \% \text { (ou seja, } 85,70,55,40,25 \text {, } \\
25 \% \text { durante os dias de afunilamento } 1 \text { a } 6 \text {, } \\
\text { respectivamente). Teste de performance: Os } \\
\text { sujeitos realizaram duas corridas competitivas } \\
\text { de } 800 \text { m com } 7 \text { dias de diferença, antes e } \\
\text { depois da redução. Os atletas competiram em } \\
\text { três grupos de pacientes submetidos à VMT } \\
\text { (conicidade de volume moderado) e LVT } \\
\text { (conicidade de baixo volume) durante as três } \\
\text { semanas anteriores à conicidade (treinamento } \\
\text { normal) e durante cada dia de conicidade. }\end{array}$ & \\
\hline $\begin{array}{l}\text { SANTOS- } \\
\text { CONCEJERO et } \\
\text { al. } 2017\end{array}$ & $\begin{array}{l}\text { Estudo experimental com } \\
15 \text { corredores de elite } \\
\text { quenianos da tribo } \\
\text { Kalenjin com média de } \\
\text { idade de } 23.7 \pm 4.2 \text {. }\end{array}$ & $\begin{array}{l}\text { Analisar a resposta da oxigenação } \\
\text { cerebral durante o treinamento } \\
\text { intervalado de alta intensidade e sua } \\
\text { relação com o desempenho na corrida }\end{array}$ & $\begin{array}{l}\text { Todos atletas completaram uma sessão de } 5 \mathrm{~km} \\
\text { na esteira antes do experimento. } \\
\text { Foram } 3 \text { testes em } 3 \text { dias consecutivos. 1- } 5 \mathrm{~km} \text {; } \\
\text { 2- um teste máximo de } \mathrm{VO} 2 \text { máx.; } 3 \text { - um teste } \\
\text { de treino fatigante (O treino consiste de } \\
\text { repetidas corridas de } 1 \mathrm{Km} \text { em um ritmo } 5 \% \\
\text { mais rápido que o respectivo } 5 \mathrm{Km} \\
\text { completados } 2 \text { dias antes com } 30 \mathrm{~s} \text { de } \\
\text { recuperação entre as repetições. Contra-relógio } \\
\text { de } 5 \text { km e um teste de treinamento para fadiga } \\
\text { em uma esteira (repetidas corridas de } 1 \mathrm{~km} \text { a } \\
\text { um ritmo } 5 \% \text { mais rápido do que o ritmo médio }\end{array}$ & $\begin{array}{l}\text { Demonstrou um declínio na oxigenação cerebral em } \\
\text { um grupo de elite de corredores quenianos durante } \\
\text { uma sessão típica e fatigante do HIIT. Os atletas } \\
\text { não oxigenam tão bem quando forçados a exercer } \\
\text { seus limites fisiológicos podendo diminuir o } \\
\text { rendimento. Os atletas mais bem-sucedidos foram } \\
\text { capazes de suportar quedas maiores na oxigenação } \\
\text { cerebral. }\end{array}$ \\
\hline
\end{tabular}


Research, Society and Development, v. 10, n. 5, e24310515008, 2021

(CC BY 4.0) | ISSN 2525-3409 | DOI: http://dx.doi.org/10.33448/rsd-v10i5.15008

\begin{tabular}{|c|c|c|c|c|}
\hline & & & $\begin{array}{l}\text { de } 5 \mathrm{~km} \text { com uma recuperação de } 30 \text { s até a } \\
\text { exaustão. }\end{array}$ & \\
\hline $\begin{array}{l}\text { SCHOENMAKE } \\
\text { et al } 2018\end{array}$ & $\begin{array}{l}\text { Estudo experimental com } \\
12 \quad \text { corredores } \\
\text { recreacionais treinados do } \\
\text { sexo masculino com } \\
\text { idade média de } 34 \pm 11 \\
\text { anos. }\end{array}$ & $\begin{array}{l}\text { Examinar os efeitos de diferentes } \\
\text { durações de recuperação nas velocidades } \\
\text { de corrida auto selecionadas, respostas } \\
\text { fisiológicas e classificações de esforço } \\
\text { percebido (EPR) em um protocolo de } \\
\text { treinamento intervalado de alta } \\
\text { intensidade (HIIT) comumente utilizado. }\end{array}$ & $\begin{array}{l}\text { Cinco visitas no laboratório. Na primeira, } \\
\text { realizaram um teste de esforço incremental em } \\
\text { esteira para determinar a captação máxima de } \\
\text { oxigênio (VO2max) e a frequência cardíaca } \\
\text { (FCmax). O teste iniciou-se a } 8 \mathrm{~km} / \mathrm{h} \text {, } \\
\text { aumentando } 1 \mathrm{~km} / \mathrm{h} \text { a cada minuto até a } \\
\text { exaustão ou quando pelo menos dois dos } \\
\text { seguintes critérios foram atendidos: (1) FC } \geq \\
\text { 90\% do máximo previsto pela idade; (2) razão } \\
\text { de troca respiratória (RER) } \geq 1,10 ; \text { (3) } \\
\text { estabilidade do VO2, apesar do aumento da } \\
\text { intensidade. Em quatro visitas subsequentes, os } \\
\text { participantes realizaram uma sessão HIIT } \\
\text { compreendendo seis intervalos de trabalho de } 4 \\
\text { minutos, nos quais a duração da recuperação } \\
\text { entre os intervalos de trabalho era igual a uma } \\
\text { duração fixa (1MIN, 2MIN, 3MIN) ou uma } \\
\text { duração auto selecionada (ssMIN). Nos } \\
\text { intervalos de recuperação, os participantes } \\
\text { estavam livres para escolher caminhar ou ficar } \\
\text { de pé. }\end{array}$ & $\begin{array}{l}\text { As respostas da percepção de esforço foram } \\
\text { semelhantes entre os protocolos, mostrando um } \\
\text { aumento gradual a cada intervalo progressivo do } \\
\text { treinamento. No entanto, as velocidades de corrida } \\
\text { foram maiores quando os participantes receberam o } \\
\text { maior período de recuperação ( } 3 \text { min). Durações de } \\
\text { recuperação mais longas podem facilitar uma carga } \\
\text { de treinamento externa mais alta (corrida mais } \\
\text { rápida), mantendo uma carga de treinamento } \\
\text { interna semelhante (estímulo fisiológico) e, } \\
\text { portanto, permitir maiores adaptações ao } \\
\text { treinamento. }\end{array}$ \\
\hline
\end{tabular}

Fonte: Autores. 


\section{Discussão}

Este trabalho promoveu uma revisão na literatura em busca de estudos que abordavam os mecanismos que poderiam atrapalhar o desempenho de corredores provocados pelo treinamento intervalado de alta intensidade em praticantes de corrida, e então poder discutir sobre os possíveis mecanismos que levariam a tais lesões. Foram localizados poucos estudos que se adequavam a proposta do trabalho; e ainda apresentavam amostra reduzida o que reduz a qualidade dos estudos e não permite respostas conclusivas sobre o tema.

$\mathrm{O}$ aumento de lactato muscular e a diminuição das reservas de fosfato levam a fadiga muscular e parecem estar associados a mudanças em alguns padrões de movimento, ocasionando movimentos inadequados que costumam estar associados ao aumento no risco do aparecimento de algumas lesões encontradas na corrida (García-Pinillos, Molina-Molina, Párraga-Montilla, \& Latorre-Román, 2019). A fadiga muscular pode causar alteração biomecânica e neuromuscular, levando ao desequilíbrio muscular e consequentemente instabilidade articular, além de prejuízos no desempenho da prática esportiva (Latorre-Román et al., 2017). A corrida com intensidade mais elevada, requer ativação de mais unidades motoras e maior recrutamento de fibras musculares oxidativas e glicolíticas, além disso, o aumento no metabolismo muscular inibe a atividade de certas enzimas como as ATPases, bomba de sódio e potássio, cálcio e miosinas, interferindo na contração muscular. O aumento da velocidade, leva ao maior impacto articular nos membros inferiores e maior comprometimento neuromuscular, principalmente dos isquiotibiais (García-Pinillos, Soto-Hermoso, \& Latorre-Román, 2016; Latorre-Román et al., 2017).

Porém um estudo analisou que uma sessão de HIIT com corredores de endurance não perturba consistentemente ou substancialmente a cinemática da corrida de corredores treinados do sexo masculino. Além disso, nem o desempenho atlético nem o nível de exaustão atingido parecem ser determinantes na resposta cinemática durante um treino de HIIT (García-Pinillos et al., 2016). Corroborando com outro artigo do mesmo autor que constatou que a maior intensidade associada ao consumo máximo de oxigênio não perturba a cinemática da corrida de corredores de resistência treinados (García-Pinillos et al., 2019). Tais achados são evidenciados por outro estudo que comparou treze atletas de elite altamente treinados, e avaliou as condições fatigadas e não fatigadas no início e no final do protocolo respectivamente, e concluiu que não houve alterações nas características cinemáticas e dos padrões de pisada (Latorre-Román et al., 2017).

O risco de lesão pode também estar associado à natureza repetitiva da corrida e às forças de reação do solo causadas pelo impacto durante a corrida e o intervalo de descanso ativo ou passivo entre os tiros no treinamento com o HIIT reduziriam essa fadiga ao permitir um menor desgaste muscular (Latorre-Román et al., 2017). O tempo de recuperação pode ser uma estratégia interessante, pois durações de recuperação mais longas podem facilitar uma carga de treinamento externa mais alta (velocidade de corrida) enquanto mantem uma carga interna semelhante (estímulo fisiológico) nas sessões HIIT e, portanto, podem permitir maiores adaptações de treinamento reduzindo lesões. Intervalo de recuperação mais longo ( 3 min) entre esforços, facilita uma velocidade de corrida mais rápida, o que é particularmente importante quando o foco da sessão é o trabalho de velocidade (Schoenmakers \& Reed, 2019).

Grande parte das lesões na corrida são atribuídas ao overuse (García-Pinillos et al., 2019, 2016) porém são necessárias mais evidências sobre os efeitos cumulativos utilizando HIIT nas sessões do treino de corrida (Latorre-Román et al., 2017). Lesões podem estar relacionadas a cargas de treinamento elevadas, por isso, a manipulação correta da intensidade, duração e frequência do treinamento são importantes para que ocorra as adaptações cardiovasculares e neuromusculares desejadas com segurança (Owen et al., 2015). Visto que já é sabido que lesões por uso excessivo representam $49 \%$ de problemas para os atletas (Clarsen et al., 2014). Ainda, corredores de média distância podem reduzir progressivamente o volume habitual de treinamento em pelo menos 75\% que não apresentarão sinais de destreinamento (Mujika et al., 2000).

Outro fator que podemos considerar, foi exposto no estudo que analisou 15 corredores quenianos de elite para 
entender a resposta da oxigenação cerebral durante o treinamento intervalado de alta intensidade e sua relação com o desempenho na corrida, concluiu-se que os atletas não oxigenam tão bem quando forçados a exercer seus limites fisiológicos podendo diminuir seu rendimento e se predisporem a lesões (Santos-Concejero et al., 2017).

O treinamento intervalado de alta intensidade (HIIT) induz adaptações metabólicas e de desempenho do músculo esquelético que se assemelham ao treinamento de resistência tradicional, apesar de um baixo volume total de exercícios (Little et al., 2010). É recomendado que o HIIT e a corrida contínua (CR) façam parte de programas de treinamento para corredores de resistência maximizando assim as adaptações ao treinamento. Além disso, 2 a 3 sessões de HIIT, trabalhando próximo ou acima do VO2máx na sessão, permitem melhora do desempenho em corredores de endurance. Uma boa prática utilizando o HIIT para treinamento dos corredores de resistência incluiria protocolos envolvendo curtos períodos de trabalho (<1 min.) com relações entre trabalho e descanso de aproximadamente 1: 1 a 1: 2, sendo executados com intensidades próximo a 100\% do VO2máx. A carga de trabalho baseada no HIIT varia de acordo com a periodização do treinamento, que deve basear-se no princípio progressivo de sobrecarga (García-Pinillos et al., 2017). Treinadores devem programar uma periodização adequada, levando em consideração tanto a intensidade quanto o volume dos treinos e que treinamentos com duração longa devem ser evitados (Owen et al., 2015).

A implementação de medidas preventivas implica uma mudança ou modificação do comportamento do atleta e, na maioria dos casos, de outros envolvidos nos cuidados e contexto próximos ao atleta, como por exemplo, o treinador (Verhagen \& van Nassau, 2019). A principal dificuldade dos treinadores e atletas é administrar a relação de carga e volume absoluto de treinamento, e a possibilidade de modificar algumas variáveis sem que haja impacto fisiológico e neuromuscular de forma perigosa para o atleta (García-Pinillos et al., 2019).

Durante a pré-temporada ocorrem mais lesões se comparados a outras temporadas (Little et al., 2010). Isso acontece, devido ao aumento da intensidade e do volume de treinamento. Sendo necessário que os profissionais que cercam o atleta, escolham qual é a melhor forma e mais segura de se treinar para atingir a melhor performance e correndo menos risco de lesões (Owen et al., 2015). Algumas estratégias podem ser utilizadas para auxiliar na forma mais segura e eficiente de controlar essa intensidade e o volume, como por exemplo as zonas de treino para controle do sistema cardíaco, o GPS (Sistema de posicionamento global), alguns questionários e o próprio relato dos atletas (Owen et al., 2015). Para determinar a gravidade das lesões, podemos analisar: a natureza da lesão, duração, atenção médica, tempo afastado da atividade ou do esporte, tempo afastado do trabalho, dano permanente e o custo da lesão (Hespanhol Junior et al., 2015). Ao utilizar sistemas de monitoramento confiáveis e aplicar de maneira contínua e a longo prazo, será possível identificar o ônus das lesões, identificar os possíveis casos em um estágio inicial, implementar intervenções precoces e gerar dados para prevenção de lesões esportivas, sendo a implementação de sistemas de monitoramento de lesões esportivas fortemente recomendada (Hespanhol Junior et al., 2015).

É importante implementar medidas preventivas, que impliquem em mudanças ou modificações no corredor e no caso dos corredores profissionais deve-se incluir os envolvidos com esses atletas, como o técnico e sua comissão (Verhagen \& van Nassau, 2019). As lesões podem ser uma barreira para essa prática, podendo gerar custos e atraso na volta ao esporte o que torna importante encontrar formas de preveni-las (Hespanhol Junior et al., 2015).

\section{Conclusão}

Ainda há poucos estudos que investigaram a ocorrência de lesões com a prática do treinamento intervalado de alta intensidade em corredores. Dos estudos encontrados, as amostras eram pequenas e apresentavam amostras diversas das diferentes práticas de corrida como corredores de elite e recreativos. 
Novos estudos com maior rigor metodológico devem ser realizados para uma melhor investigação do risco de lesões na prática do HIIT em corredores. Algo que deve ser notado é a grande variação de protocolos de HIIT encontrados nos estudos. Porém vale destacar que esta modalidade de treinamento apresenta estrutura com períodos de repouso ativo ou passivo entre os tiros, volume menor de treinamento e sua determinação de intensidade é baseada nos próprios parâmetros do indivíduo fazendo com que o risco de lesões possa ser reduzido.

\section{Referências}

Bucci, M., Vinagre, E. C., Campos, G. E. R., Curi, R., \& Pithon-Curi, T. C. (2005). Efeitos do treinamento concomitante hipertrofia e endurance no músculo esquelético. Rev. Bras. Ciênc. Mov, 13(1), 17-28. https://doi.org/10.18511/rbcm.v13i1.608

Buchheit, M., \& Laursen, P. B. (2013). High-Intensity Interval Training, Solutions to the Programming Puzzle. Sports Medicine, 43(5), 313-338. https://doi.org/10.1007/s40279-013-0029-x

Burgomaster, K. A., Hughes, S. C., Heigenhauser, G. J. F., Bradwell, S. N., \& Gibala, M. J. (2005). Six sessions of sprint in terval training increases muscle oxidative potential and cycle endurance capacity in humans. Journal of Applied Physiology, 98(6), 1985-1990. https://doi.org/10.1152/japplphysiol.01095.2004

Clarsen, B., Rønsen, O., Myklebust, G., Flørenes, T. W., \& Bahr, R. (2014). The Oslo Sports Trauma Research Center questionnaire on health problems: a new approach to prospective monitoring of illness and injury in elite athletes. British Journal of Sports Medicine, 48(9), 754-760. https://doi.org/10.1136/bjsports-2012-092087

Freitas, D. A., Rocha-Vieira, E., De Sousa, R. A. L., Soares, B. A., Rocha-Gomes, A., Chaves Garcia, B. C., \& Leite, H. R. (2019). High-intensity interval training improves cerebellar antioxidant capacity without affecting cognitive functions in rats. Behavioural Brain Research, 376 , 112181. https://doi.org/10.1016/j.bbr.2019.112181

García-Pinillos, F., Molina-Molina, A., Párraga-Montilla, J. A., \& Latorre-Román, P. A. (2019). Kinematic alterations after two high-intensity intermittent training protocols in endurance runners. Journal of Sport and Health Science, 8(5), 442-449. https://doi.org/10.1016/j.jshs.2016.11.003

García-Pinillos, F., Soto-Hermoso, V. M., \& Latorre-Román, P. A. (2017). How does high-intensity intermittent training affect recreational endurance runners? Acute and chronic adaptations: A systematic review. Journal of Sport and Health Science, 6(1), 54-67. https://doi.org/10.1016/j.jshs.2016.08.010

García-Pinillos, F., Soto-Hermoso, V. M., \& Latorre-Román, P. Á. (2016). Do Running Kinematic Characteristics Change over a Typical HIIT for Endurance Runners? Journal of Strength and Conditioning Research, 30(10), 2907-2917. https://doi.org/10.1519/JSC.0000000000001380

Gibala, M. J., \& McGee, S. L. (2008). Metabolic Adaptations to Short-term High-Intensity Interval Training. Exercise and Sport Sciences Reviews, 36(2), 5863. https://doi.org/10.1097/JES.0b013e318168ec1f

Gillen, J. B., \& Gibala, M. J. (2014). Is high-intensity interval training a time-efficient exercise strategy to improve health and fitness? Applied Physiology, Nutrition, and Metabolism, 39(3), 409-412. https://doi.org/10.1139/apnm-2013-0187

Kohn, T. A. et al, Specific muscle adaptations in type II fibers after high-intensity interval training of well-trained runners. Scand J Med Sci Sports 2011: 21: 765-772. https://pubmed.ncbi.nlm.nih.gov/20492589.

Hespanhol Junior, L. C., Barboza, S. D., van Mechelen, W., \& Verhagen, E. (2015). Measuring sports injuries on the pitch: a g uide to use in practice. Brazilian Journal of Physical Therapy, 19(5), 369-380. https://doi.org/10.1590/bjpt-rbf.2014.0110

Latorre-Román, P. Á., García Pinillos, F., Bujalance-Moreno, P., \& Soto-Hermoso, V. M. (2017). Acute effects of high-intensity intermittent training on kinematics and foot strike patterns in endurance runners. Journal of Sports Sciences, 35(13), 1247-1254. https://doi.org/10.1080/02640414.2016.1218038

Little, J. P., Safdar, A., Wilkin, G. P., Tarnopolsky, M. A., \& Gibala, M. J. (2010). A practical model of low-volume high-intensity interval training induces mitochondrial biogenesis in human skeletal muscle: potential mechanisms. The Journal of Physiology, 588(6), 1011-1022. https://doi.org/10.1113/jphysiol.2009.181743

MacInnis, M. J., \& Gibala, M. J. (2017). Physiological adaptations to interval training and the role of exercise intensity. The Journal of Physiology, 595(9), 2915-2930. https://doi.org/10.1113/JP273196

Melo, C. S., Rocha-Vieira, E., Freitas, D. A., Soares, B. A., Rocha-Gomes, A., Riul, T. R., \& Leite, H. R. (2019). A single session of high-intensity interval exercise increases antioxidants defenses in the hippocampus of Wistar rats. Physiology and Behavior, 211. https://doi.org/10.1016/j.physbeh.2019.112675

Mujika, I., Goya, A., Padilla, S., Grijalba, A., Gorostiaga, E., \& Ibañez, J. (2000). Physiological responses to a 6-d taper in middle-distance runners: Influence of training intensity and volume. Medicine and Science in Sports and Exercise, 32(2), 511-517. https://doi.org/10.1097/00005768-200002000-00038

Owen, A. L., Forsyth, J. J., Wong, D. P., Dellal, A., Connelly, S. P., \& Chamari, K. (2015). Heart Rate-Based Training Intensity and Its Impact on Injury Incidence Among Elite-Level Professional Soccer Players. Journal of Strength and Conditioning Research, 29(6), 1705-1712. https://doi.org/10.1519/JSC.0000000000000810

Santos-Concejero, J., Billaut, F., Grobler, L., Oliván, J., Noakes, T. D., \& Tucker, R. (2017). Brain oxygenation declines in elite Kenyan runners during a maximal interval training session. European Journal of Applied Physiology, 117(5), 1017-1024. https://doi.org/10.1007/s00421-017-3590-4 
Research, Society and Development, v. 10, n. 5, e24310515008, 2021

(CC BY 4.0) | ISSN 2525-3409 | DOI: http://dx.doi.org/10.33448/rsd-v10i5.15008

Schoenmakers, P. P. J. M., \& Reed, K. E. (2019). The effects of recovery duration on physiological and perceptual responses of trained runners during four self-paced HIIT sessions. Journal of Science and Medicine in Sport, 22(4), 462-466. https://doi.org/10.1016/j.jsams.2018.09.230

Verhagen, E., \& van Nassau, F. (2019). Implementation science to reduce the prevalence and burden of MSK disorders following sport and exercise-related injury. Best Practice \& Research Clinical Rheumatology, 33(1), 188-201. https://doi.org/10.1016/j.berh.2019.02.011 